\title{
EXTRAÇÕES SEQUENCIAIS DE CHUMBO E ZINCO EM SOLOS DE ÁREA DE MINERAÇÃO E METALURGIA DE METAIS PESADOS ${ }^{(1)}$
}

\author{
Larissa Kummer ${ }^{(2)}$, Vander de Freitas Melo( ${ }^{(3)}$, Yara Jurema \\ Barros $^{(4)}$ \& Julio César Rodrigues de Azevedo ${ }^{(5)}$
}

\begin{abstract}
RESUMO
A extração sequencial permite obter informações mais detalhadas sobre origem, modo de ocorrência, disponibilidade biológica e físico-química, mobilização e transporte dos metais pesados em ambientes naturais. Com o objetivo de estudar as formas de $\mathrm{Pb}$ e $\mathrm{Zn}$ foram selecionados oito perfis de solos de diferentes locais dentro de área de mineração e metalurgia de $\mathrm{Pb}$, no município de Adrianópolis (PR), Vale do Rio Ribeira. As amostras foram coletadas nas profundidades de 0 a 10,10 a 20 e 20 a $40 \mathrm{~cm}$. Foram determinados os teores totais de $\mathrm{Pb}$ e $\mathrm{Zn}$ e sua especiação nas formas: solúvel; trocável; ligada a carbonatos; ligada à matéria orgânica; ligada aos óxidos de Fe de $\mathrm{Al}$ de baixa cristalinidade; ligada aos óxidos de Al cristalinos e filossilicatos 1:1 e 2:1; e residual. Com base nos valores percentuais de participação de cada fração nos teores totais, procedeu-se ao agrupamento das amostras similares por meio da análise de componentes principais (PCA). Houve basicamente duas formas de contaminação dos perfis de solo, sendo uma em decorrência das partículas das chaminés e a outra em função do acúmulo de rejeitos sólidos sobre os solos. A primeira foi mais prejudicial ao ambiente em razão dos maiores teores totais e de formas mais disponíveis no solo (solução mais trocável). De modo geral, houve maior associação de $\mathrm{Pb}$ aos carbonatos, seguida das frações residual e óxidos de $\mathrm{Fe}$ e $\mathrm{Al}$ de baixa cristalinidade. $\mathrm{O} \mathrm{Zn}$ apresentou-se em formas mais insolúveis, aumentando a participação da fração residual nos teores totais. A $\mathrm{PCA}$ foi sensível às diferentes formas de $\mathrm{Pb}$ nos solos, pois promoveu o agrupamento das amostras, principalmente, em função da participação das formas trocáveis, ligadas aos óxidos de Fe e Al e ligadas aos carbonatos, em relação aos teores totais.

Termos de indexação: especiação de metais pesados, formas solúveis, adsorção específica, adsorção não específica.
\end{abstract}

\footnotetext{
(1) Recebido para publicação em 1 de setembro de 2010 e aprovado em 15 de setembro de 2011.

(2) Professora do Curso de Engenharia Ambiental, Universidade Tecnológica Federal do Paraná - UTFPR. Linha Santa Bárbara s/n, CEP 85601-971 Francisco Beltrão (PR). E-mail: lkummer@utfpr.edu.br

(3) Professor do Departamento de Solos e Engenharia Agrícola, Universidade Federal do Paraná - UFPR. Rua dos Funcionários 1540, CEP 80035-050 Curitiba (PR). Bolsista CNPq. E-mail: vanderfm@ufpr.br

(4) Mestre em Ciência do Solo, UFPR. E-mail: yarajuba@gmail.com

(5) Professor do Departamento de Química e Biologia, Universidade Tecnológica Federal do Paraná - UTFPR. Curitiba (PR). E-mail: jcrazevedo@hotmail.com
} 


\title{
SUMMARY: SEQUENTIAL EXTRACTION OF LEAD AND ZINC FROM SOILS OF HEAVY METAL MINING AND PROCESSING AREA
}

\begin{abstract}
Sequential extraction allows more detailed information about the origin of heavy metals in natural environments, their mode of occurrence, biological and physico-chemical availability, mobilization and transport. To study the $\mathrm{Pb}$ and $\mathrm{Zn}$ forms, eight soil profiles were selected at different sites within a $\mathrm{Pb}$ mining and metallurgy area in the city of Adrianópolis, state of Paraná, in the Rio Ribeira valley. Samples were collected from the layers 00-10, 10-20 and $20-40 \mathrm{~cm}$. The total $\mathrm{Pb}$ and $\mathrm{Zn}$ contents and their speciation in the forms: soluble, exchangeable, carbonate-bound, bound to organic matter, bound to $\mathrm{Fe}$ and Al oxides of low crystallinity; linked to crystalline Al oxides and phyllosilicates 1:1 and 2:1; and residual. Based on the percentage values of each fraction in the total contents, similar samples were grouped by principal component analysis (PCA). There were basically two contamination forms of soil profiles; the first was the result of particles from the chimneys and the second due to the accumulation of solid waste on the soil surface. The first was more environmentally damaging due to the higher total and soluble forms in the soil (in the exchangeable and in the solution forms). Overall, there was a greater association of $\mathrm{Pb}$ with carbonates, and residual fractions followed by $\mathrm{Fe}$ and $\mathrm{Al}$ oxides of low crystallinity. Zinc was found in more insoluble forms, increasing the participation in total contents of the residual fraction. The PCA is sensitive to different $\mathrm{Pb}$ forms in the soils, underlying the grouping of samples, mainly based on the participation of exchangeable forms, bound to Fe and Al oxides and to carbonates, in relation to the total content.
\end{abstract}

Index terms: speciation of heavy metals, soluble forms, specific adsorption, non-specific adsorption.

\section{INTRODUÇÃO}

As atividades de mineração e metalurgia de metais pesados podem contribuir diretamente e de maneira significativa na contaminação do ambiente por meio da emissão de fumaças contendo elementos metálicos e também pela deposição de rejeitos diretamente ao solo (Bosso \& Enzweiler, 2008). Concentrações elevadas de metais pesados no solo podem interferir no ambiente, alterando a produtividade, biodiversidade e sustentabilidade dos ecossistemas, além de ocasionar riscos aos seres humanos e animais (Sun et al, 2001; Kede et al., 2008). O potencial de dano desses elementos agrava-se quando eles são absorvidos pelas plantas e quando sua quantidade excede a máxima capacidade de retenção do solo, tornando-se facilmente lixiviáveis, com consequente aporte em águas subterrâneas (Pandolfo et al., 2008).

No município de Adrianópolis (PR), por mais de 50 anos, foram desenvolvidas as atividades de mineração e metalurgia de primeira fusão de $\mathrm{Pb} . \mathrm{O}$ mineral de interesse econômico na área foi a galena (PbS), com ocorrência de veios do mineral nas rochas da região (Oliveira et al., 2002). A jazida no município de Adrianópolis (Panelas de Brejaúvas) foi responsável por cerca de $55 \%$ da produção de $\mathrm{Pb}$ do Vale do Rio Ribeira; foram extraídos aproximadamente $1.330 .000 \mathrm{t}$ de minério, com teor médio de $\mathrm{Pb}$ de $69 \mathrm{~g} \mathrm{~kg}^{-1}$ (Moraes, 1997). Em 1995 a mineradora encerrou suas atividades, deixando a céu aberto, sem nenhuma proteção, cerca de 177.000 t de resíduos de beneficiamento de $\mathrm{Pb}$ (Eysink, 1988).
Estudos mais recentes demonstraram que os efeitos da mineração e metalurgia de $\mathrm{Pb}$ ainda se faziam presentes. Destaca-se o trabalho de Cunha (2003), que avaliou o sangue de 335 crianças em idade escolar (7 a 14 anos), entre 1999 e 2000, que moravam no entorno da antiga área de mineração (Vila Mota). Aproximadamente, $70 \%$ das crianças apresentaram teores de $\mathrm{Pb}$ três vezes superiores aos limites preconizados pelo U.S. Center for Disease Control (CDC) e Organização Mundial de Saúde (OMS). Nos trabalhos de Barros et al. (2010a,b) ficou demonstrado que solos com maiores teores de $\mathrm{Pb}$ na área da mineração apresentaram menor população e atividade microbiana e de organismos da mesofauna edáfica. Andrade (2009) encontrou teores de $\mathrm{Pb}$ nas águas coletadas nas redes de drenagens da área acima do limite permitido pela Resolução Conama 357/05, e os teores de $\mathrm{Pb}$ nos sedimentos atingiram $24.300 \mathrm{mg} \mathrm{kg}^{-1}$.

Os critérios para o controle da poluição do solo baseiam-se geralmente na quantidade total de metais pesados, que fornece os dados de sua reserva no solo (Camargo et al., 2000). No entanto, o teor total não é característica que expresse a disponibilidade para as plantas e os organismos. A distribuição dos metais nos diversos sítios de adsorção das partículas reativas do solo pode ser usada para estimar a sua biodisponibilidade ou predizer contaminações do ambiente (Bosso \& Enzweiler, 2008; Casali et al., 2008). Esse tipo de estudo pode ser realizado com o uso da técnica de extração sequencial, também chamada de fracionamento químico de metais (Shuman, 1979; Sposito et al., 1982; Mattiazzo et al., 
2001). A extração sequencial permite obter informações mais detalhadas sobre origem, modo de ocorrência, disponibilidade biológica e físico-química, mobilização e transporte dos metais em ambientes naturais (Tessier et al., 1979).

A extração sequencial inicia-se com extrator fraco, como a água, e termina com extrator forte, como o ácido concentrado. O tempo de extração e a relação solo-solução permitem solubilizar a fração específica do elemento da fase sólida, minimizando a interferência nas demais frações (Kotas \& Stasicka, 2000; Casali et al., 2008). Tem-se verificado que os elementos de maior mobilidade encontram-se, predominantemente, nas formas químicas solúvel, trocável e ligados a carbonato, enquanto os de baixa mobilidade aparecem ligados às frações óxidos de $\mathrm{Fe}$, Mn, além das frações orgânica e residual (Amaral Sobrinho et al., 1994). Apesar das vantagens do processo de extrações sequenciais em relação apenas à determinação dos teores totais de metais pesados no solo, podem ocorrer problemas de baixa seletividade dos extratores (Shan \& Chen, 1993; Shiowatana et al., 2001), redistribuição dos metais durante os processos de extração (Tu et al., 1994; Rakasasalaya et al., 1996) e dependência dos resultados das condições operacionais (Sahuquillo et al., 1999).

Este trabalho teve como objetivo determinar as formas de $\mathrm{Pb}$ e $\mathrm{Zn}$ em solos de área de mineração e metalurgia de metais pesados, no município de Adrianópolis (PR), por meio de extrações químicas sequenciais, aplicando-se o controle de perda de massa das amostras pelos tratamentos. Procurou-se, também, relacionar os diferentes ambientes de contaminação da área com os teores e as formas desses poluentes nos solos.

\section{MATERIAL E MÉTODOS}

\section{Área de estudo e amostragem dos solos}

O estudo foi desenvolvido a partir de oito locais de amostragem de solos de área de mineração e metalurgia de $\mathrm{Pb}$ (Figura 1), no município de Adrianópolis (PR). A compartimentalização da paisagem em unidades mais homogêneas, com menor variabilidade das classes de solos, usando o geoprocessamento, e a identificação de diferentes ambientes em função da forma de contaminação possibilitaram a amostragem mais representativa da área sob influência direta das atividades de mineração e metalurgia de $\mathrm{Pb}$ (linha mais espessa na Figura 1). Os critérios para seleção dos locais de amostragem dos solos foram apresentados por Andrade et al. (2009) e suas características estão no quadro 1.

As amostras de solo, coletadas nas camadas de $0-$ 10, 10-20 e 20-40 cm de profundidade, foram secas

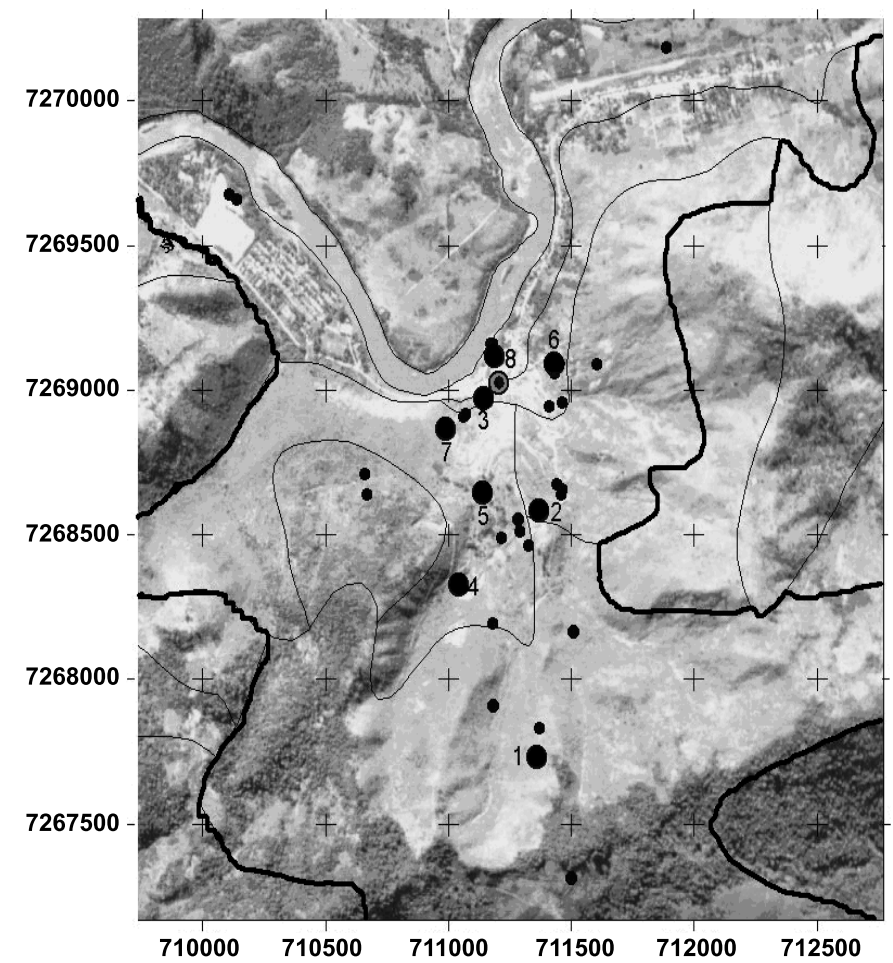

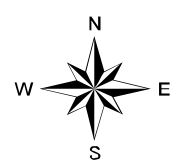

Legenda:

- Pontos de amostragem

Pontos de amostragem selecionados para estudo

Fábrica desativada

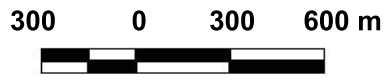

Base cartográfica: Carta de Apiaí, IBGE, Secretaria de Planejamento da Presidência da República.

Sistema de Projeção: Universal Transversa de Mercator. Datum: SAD-69.

Figura 1. Foto aérea georreferenciada com os compartimentos geomorfológicos (linhas mais finas), localização da fábrica abandonada e locais de coleta (1 a 8) dentro da área selecionada para o estudo (linha mais espessa). Os pontos menores representam a coleta prévia de solos para reconhecimento da área e definição dos ambientes em função das diferentes formas de contaminação, conforme detalhes apresentados por Andrade et al. (2009). 
ao ar e passadas em peneira com malha de $2 \mathrm{~mm}$ (TFSA). A textura e as características químicas da TFSA (métodos descritos em Embrapa, 1997; Kummer et al., 2010) e a mineralogia da fração argila (métodos apresentados por Melo et al., 2001, 2002; Kummer et al., 2010) das amostras são apresentadas nos quadros 2 e 3 , respectivamente.

\section{Determinação dos teores totais de $\mathbf{P b}$ e $\mathbf{Z n}$}

A digestão das amostras de solo foi realizada em forno de micro-ondas, colocando-se $0,25 \mathrm{~g}$ de solo, previamente seco a $40{ }^{\circ} \mathrm{C}$ por $12 \mathrm{~h}$, na presença de $4 \mathrm{~mL}$ de $\mathrm{HNO}_{3}$ e $3 \mathrm{~mL}$ de $\mathrm{HF}$ concentrados e $1 \mathrm{~mL}$ de $\mathrm{H}_{2} \mathrm{O}_{2}(30 \% \mathrm{v} / \mathrm{v})$ (Lim \& Jackson, 1986). Os frascos foram submetidos a aquecimento por $10 \mathrm{~min}$ a uma potência de $1.000 \mathrm{~W}$ até atingirem temperatura de $200 \pm 2{ }^{\circ} \mathrm{C}$, a qual foi mantida por mais $20 \mathrm{~min}$. Após 30 min de resfriamento das amostras no interior do aparelho, a suspensão foi filtrada para separar possível material residual refratário (p. ex., óxidos de Ti). Aferiuse a massa do extrato para aproximadamente $15 \mathrm{~g}$ com água deionizada. O volume foi determinado com

Quadro 1. Localização, classificação dos solos e características dos locais de amostragem identificadas em campo

\begin{tabular}{|c|c|c|c|c|c|}
\hline \multirow[b]{2}{*}{ Solo } & \multicolumn{3}{|c|}{ UTM (22J) } & \multirow{2}{*}{\multicolumn{2}{|c|}{ Observações }} \\
\hline & $\begin{array}{l}\text { N-S (m) } \\
\text { Latitude }\end{array}$ & $\begin{array}{l}\text { E-W (m) } \\
\text { Longitude }\end{array}$ & $\begin{array}{l}\text { Altitude/ } \\
\text { Distância }^{(1)}\end{array}$ & & \\
\hline 1 & $7267313 \mathrm{~S}$ & $711502 \mathrm{~W}$ & $\stackrel{\mathrm{m}}{546 / 1.560}$ & $\begin{array}{l}\text { Neossolo } \\
\text { Litólico }\end{array}$ & $\begin{array}{l}\text { Solo de referência sob mata nativa. Graças à } \\
\text { grande distância ( } 1.700 \mathrm{~m} \text { ) e altitude do local em } \\
\text { relação à fábrica (diferença de cota de } 380 \mathrm{~m}) \\
\text { não havia evidências da influência direta das } \\
\text { atividades de mineração no solo. Material de } \\
\text { origem: rochas carbonáticas/complexos granitoides. }\end{array}$ \\
\hline 2 & 7268164 & 711513 & $326 / 563$ & $\begin{array}{l}\text { Cambissolo } \\
\text { Háplico }\end{array}$ & $\begin{array}{l}\text { Posição intermediária da encosta, com } \\
\text { evidência de deposição de colúvio mais rejeitos } \\
\text { da metalurgia vindos das partes mais altas. } \\
\text { Material de origem: rochas } \\
\text { carbonáticas/complexos granitoides. }\end{array}$ \\
\hline 3 & 7268555 & 711287 & $165 / 45$ & $\begin{array}{l}\text { Neossolo } \\
\text { Litólico }\end{array}$ & $\begin{array}{l}\text { Próximo à fábrica (Figura 1), com os horizontes } \\
\text { do solo preservados e sem incorporação de rejeitos } \\
\text { sólidos da metalurgia. Perfil na base da encosta. } \\
\text { Solo com potencial (muito próximo da fábrica e } \\
\text { localizado no sentido de caminhamento dos } \\
\text { ventos) de adição de Pb particulado, por meio da } \\
\text { emissão de fumaça pelas chaminés. Material de } \\
\text { origem: rochas carbonáticas/complexos granitoides. }\end{array}$ \\
\hline 4 & 7267907 & 711187 & $327 / 590$ & $\begin{array}{l}\text { Mistura de } \\
\text { sedimento mais } \\
\text { rejeito fino }{ }^{(2)}\end{array}$ & $\begin{array}{l}\text { Vale soterrado com pilhas de rejeitos da metalurgia, } \\
\text { misturados ao solo. Como resultado, a camada de } \\
\text { coleta de amostra não apresentava estrutura } \\
\text { e horizontes pedogenéticos. Material de origem: } \\
\text { mistura de sedimentos mais rejeitos da metalurgia. }\end{array}$ \\
\hline 5 & 7268070 & 711360 & $316 / 455$ & $\begin{array}{l}\text { Mistura de solo } \\
\text { mais r ejeito } \\
\text { grosseiro }^{(2)}\end{array}$ & $\begin{array}{l}\text { Grande ocorrência de rejeitos da metalurgia } \\
\text { na superfície e parte incorporada ao solo. } \\
\text { Como resultado, a camada de coleta de } \\
\text { amostra não apresentava estrutura e } \\
\text { horizontes pedogenéticos. Material de origem: } \\
\text { mistura de solo mais rejeitos da metalurgia. }\end{array}$ \\
\hline 6 & 7268671 & 711572 & $202 / 295$ & $\begin{array}{l}\text { Neossolo } \\
\text { Quartizarênico }\end{array}$ & $\begin{array}{l}\text { Idem solo } 3 \text {, exceto pela maior distância em } \\
\text { relação à fábrica e pelo material de origem, } \\
\text { que é quartzito. }\end{array}$ \\
\hline 7 & 7268499 & 711158 & $194 / 321$ & $\begin{array}{l}\text { Cambissolo } \\
\text { Háplico }\end{array}$ & $\begin{array}{l}\text { Idem solo } 3 \text {, exceto pela maior distância em } \\
\text { relação à fábrica. }\end{array}$ \\
\hline 8 & 7268701 & 711331 & $157 / 64$ & $\begin{array}{l}\text { Neossolo } \\
\text { Flúvico }\end{array}$ & $\begin{array}{l}\text { Faixa entre a fábrica e o rio Ribeira e relevo } \\
\text { praticamente plano. Solo com potencial de } \\
\text { contaminação pela passagem da água pluvial } \\
\text { pela fábrica, que escorre em direção ao rio } \\
\text { Ribeira. Material de origem: sedimentos } \\
\text { arenosos (fluviais). }\end{array}$ \\
\hline
\end{tabular}

\footnotetext{
(1) Distância em linha reta do ponto de amostragem à fábrica desativada. ${ }^{(2)}$ Resíduo de mineração e metalurgia de chumbo intimamente misturados ao solo; devido à falta de horizontes pedogenéticos, não foi possível classificar os perfis de solos.
} 
Quadro 2. Atributos químicos das amostras dos solos e análise textural

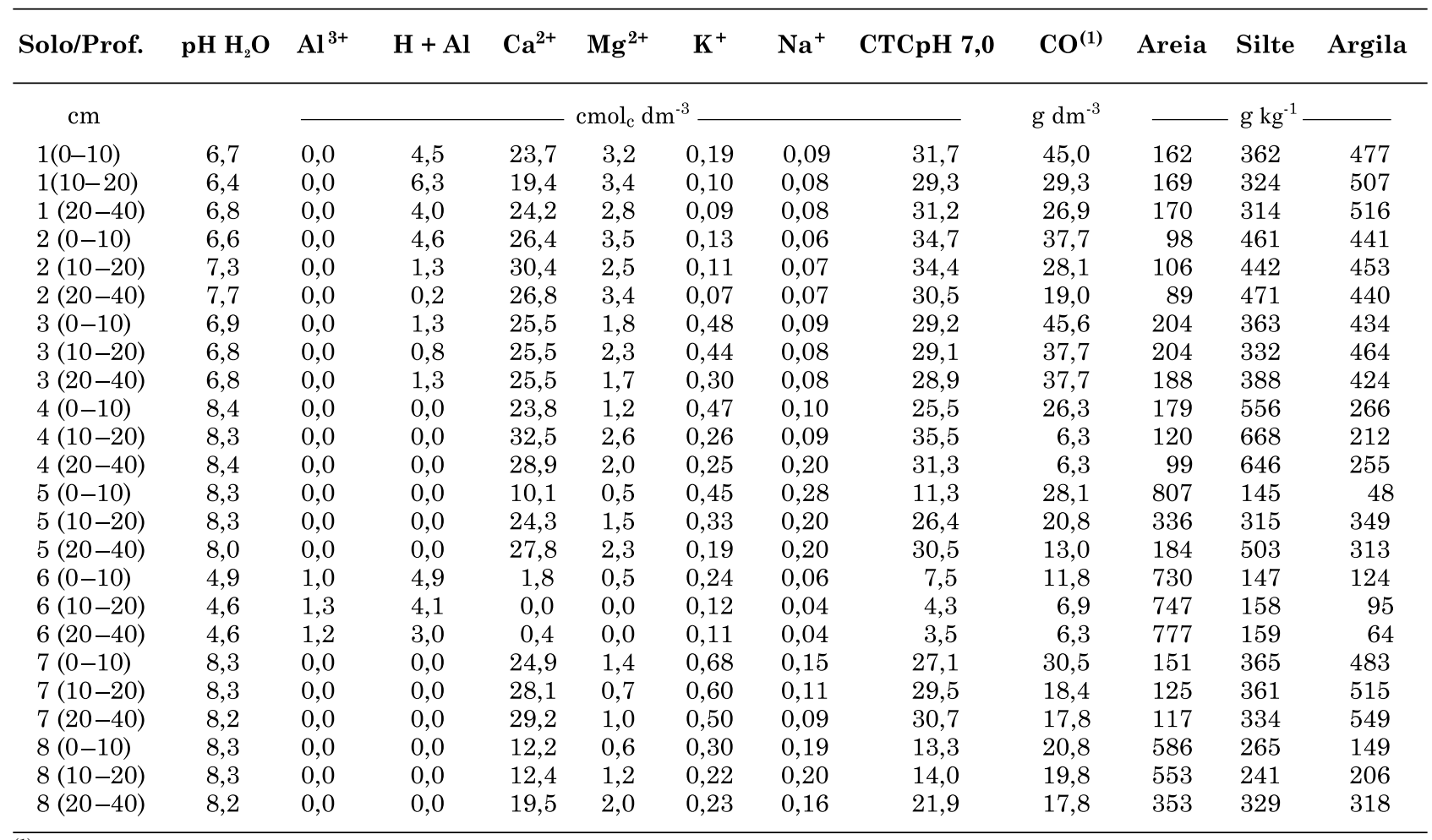

(1) CO: carbono orgânico. Fonte: Modificado de Kummer et al. (2010).

Quadro 3. Composição mineralógica da fração argila das amostras dos solos ${ }^{(1)}$

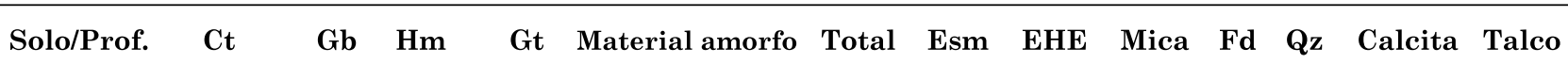

\begin{tabular}{|c|c|c|c|c|c|c|c|c|c|c|c|c|c|}
\hline $\mathrm{cm}$ & & & & $\mathrm{g} \mathrm{kg}$ & & & & & & & & & \\
\hline $1(0-10)$ & 537,4 & 35,9 & 19,7 & 7,8 & 55,6 & 656,4 & $\mathrm{nq}$ & $\mathrm{nq}$ & - & - & $\mathrm{nq}$ & - & $\mathrm{nq}$ \\
\hline $1(10-20)$ & 541,6 & 34,4 & 33,1 & 7,7 & 75,3 & 692,2 & nq & - & - & - & $\mathrm{nq}$ & - & $\mathrm{nq}$ \\
\hline $1(20-40)$ & 534,2 & 33,4 & 19,0 & 4,1 & 50,0 & 640,8 & nq & $\mathrm{nq}$ & - & - & $\mathrm{nq}$ & - & $\mathrm{nq}$ \\
\hline $2(0-10)$ & 438,6 & 51,3 & 9,7 & 21,4 & 57,8 & 578,8 & nq & nq & - & - & $\mathrm{nq}$ & - & $\mathrm{nq}$ \\
\hline $2(10-20)$ & 423,3 & 50,0 & 19,4 & 30,4 & 54,6 & 577,8 & nq & nq & - & - & nq & - & nq \\
\hline $2(20-40)$ & 441,6 & 40,0 & 10,6 & 31,8 & 44,6 & 568,6 & nq & nq & - & - & $\mathrm{nq}$ & - & $\mathrm{nq}$ \\
\hline $3(0-10)$ & 534,4 & 43,7 & 14,1 & 11,8 & 54,1 & 658,1 & nq & - & - & $\mathrm{nq}$ & $\mathrm{nq}$ & - & - \\
\hline $3(10-20)$ & 535,5 & 36,2 & 23,0 & 17,1 & 42,6 & 654,4 & nq & - & - & $\mathrm{nq}$ & $\mathrm{nq}$ & - & - \\
\hline $3(20-40)$ & 518,5 & 41,2 & 21,5 & 18,4 & 26,4 & 626,1 & nq & - & - & $\mathrm{nq}$ & $\mathrm{nq}$ & - & - \\
\hline $4(0-10)$ & 494,8 & 39,5 & 0,0 & 36,8 & 60,6 & 631,6 & nq & $\mathrm{nq}$ & $\mathrm{nq}$ & $\mathrm{nq}$ & $\mathrm{nq}$ & - & $\mathrm{nq}$ \\
\hline $4(10-20)$ & 441,3 & 29,3 & 0,1 & 27,6 & 86,2 & 584,4 & $\mathrm{nq}$ & - & 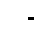 & $\mathrm{nq}$ & $\mathrm{nq}$ & - & $\mathrm{nq}$ \\
\hline $4(20-40)$ & 497,8 & 39,9 & 3,1 & 41,8 & 34,7 & 617,4 & nq & - & - & $\mathrm{nq}$ & $\mathrm{nq}$ & - & $\mathrm{nq}$ \\
\hline $5(0-10)$ & 443,4 & 35,1 & 3,2 & 37,9 & 96,9 & 616,4 & nq & - & - & - & $\mathrm{nq}$ & - & - \\
\hline $5(10-20)$ & 532,4 & 45,4 & 0,8 & 41,4 & 26,0 & 646,0 & nq & - & $\mathrm{nq}$ & - & $\mathrm{nq}$ & - & - \\
\hline $5(20-40)$ & 445,0 & 46,1 & 4,8 & 27,3 & 19,9 & 543,0 & nq & - & - & - & $\mathrm{nq}$ & $\mathrm{nq}$ & - \\
\hline $6(0-10)$ & 525,6 & 21,2 & 0,0 & 41,1 & 58,4 & 646,3 & - & - & $\mathrm{nq}$ & $\mathrm{nq}$ & $\mathrm{nq}$ & - & - \\
\hline $6(10-20)$ & 505,8 & 16,2 & 0,0 & 27,4 & 102,4 & 651,8 & - & - & $\mathrm{nq}$ & $\mathrm{nq}$ & $\mathrm{nq}$ & - & - \\
\hline $6(20-40)$ & 494,0 & 24,4 & 0,0 & 41,2 & 75,2 & 634,8 & - & - & $\mathrm{nq}$ & $\mathrm{nq}$ & $\mathrm{nq}$ & - & - \\
\hline $7(0-10)$ & 595,2 & 34,8 & 19,2 & 22,2 & 69,4 & 740,9 & $\mathrm{nq}$ & - & - & $\mathrm{nq}$ & $\mathrm{nq}$ & - & - \\
\hline $7(10-20)$ & 601,8 & 32,7 & 13,6 & 28,6 & 61,7 & 738,4 & nq & - & - & $\mathrm{nq}$ & $\mathrm{nq}$ & - & - \\
\hline $7(20-40)$ & 674,5 & 45,5 & 10,5 & 26,9 & 61,1 & 818,6 & nq & - & - & $\mathrm{nq}$ & $\mathrm{nq}$ & - & $\mathrm{nq}$ \\
\hline $8(0-10)$ & 501,8 & 32,2 & 0,0 & 38,5 & 71,9 & 644,4 & nq & $\mathrm{nq}$ & $\mathrm{nq}$ & $\mathrm{nq}$ & nq & - & - \\
\hline $8(10-20)$ & 509,1 & 40,2 & 0,0 & 45,4 & 91,1 & 685,8 & nq & nq & - & nq & nq & - & nq \\
\hline $8(20-40)$ & 538,3 & 21,5 & 7,6 & 35,1 & 79,8 & 682,2 & nq & $\mathrm{nq}$ & - & $\mathrm{nq}$ & $\mathrm{nq}$ & - & - \\
\hline
\end{tabular}

(1) Ct: caulinita, Gb: gibbsita, Hm: hematita, Gt: goethita, Material amorfo: quantidade de material extraído pelo oxalato de amônio, Esm: esmectita, EHE: esmectita com hidroxi-Al entrecamadas, Fd: feldspato, Qz: quartzo. nq: mineral apenas identificado por DRX (análise qualitativa). - ausência de reflexão por DRX. Fonte: Modificado de Kummer et al. (2010). 
base na massa e densidade do líquido. Os extratos foram analisados por espectroscopia de emissão atômica em plasma induzido (ICP-AES).

\section{Fracionamento Químico de $\mathrm{Pb}$ e $\mathrm{Zn}$}

O fracionamento químico de $\mathrm{Pb}$ e $\mathrm{Zn}$ do solo foi baseado no método descrito por Mann \& Ritchie (1993) e por Gomes et al. (1997), com modificações (Quadro 4). Após cada extração, os teores de $\mathrm{Pb}$ e $\mathrm{Zn}$ foram determinados por ICP-AES. Outros procedimentos comuns às extrações sequenciais foram: a) a massa das amostras foi tomada em balança de precisão com quatro dígitos; b) antes das pesagens, para obtenção da massa da amostra seca inicial (antes da extração) e da massa final (após a extração), as amostras foram secas em estufa a $40^{\circ} \mathrm{C}$ por $12 \mathrm{~h}$; d) lavagem do resíduo de cada extração, para remoção do excesso de sais com solução de $\left(\mathrm{NH}_{4}\right)_{2} \mathrm{CO}_{3} 0,5 \mathrm{~mol} \mathrm{~L}-1$ e com água deionizada (exceto a fração solúvel); c) durante as extrações e lavagens da amostra, a suspensão foi centrifugada a $3.500 \mathrm{rpm}$ por $10 \mathrm{~min}$; e) acidificação dos extratos com $\mathrm{HNO}_{3}$ suficiente para que o extrato final ficasse com concentração de $3 \%$, para evitar a sua deterioração; e f) extratos estocados em frascos de polietileno, para evitar a adsorção dos metais às paredes dos frascos de vidro, como alertado por Kim \& Hill (1993).

A contribuição dos metais extraídos nos diferentes tratamentos sequenciais nos teores totais de $\mathrm{Pb}$ e Zn foi calculada considerando a quantidade de material removido e os teores desses mesmos metais obtidos em cada extração sequencial. Mais detalhes sobre os cálculos podem ser obtidos em Kummer (2008). Melo et al. (2003) utilizaram o mesmo procedimento para o cálculo da contribuição de extrações sequenciais nos teores totais de K da fração argila de solos do Triângulo Mineiro.

\section{Análise estatística}

Os valores percentuais de participação de cada fase no total extraído foram então submetidos à análise dos componentes principais (PCA), utilizando o programa Statistica 6.1. A PCA é uma técnica que indica as associações entre variáveis reduzindo a dimensão do número de dados e agrupando aquelas com maior similaridade (Moita Neto \& Moita, 1998; Goloboèanin et al., 2004; Santos et al., 2008; Valladares et al., 2008). Para a apresentação dos resultados, optou-se pelo gráfico de scores e loadings no mesmo gráfico da PCA, a fim de permitir melhor visualização da influência das variáveis sobre o agrupamento das amostras. No gráfico de scores da PCA foram adicionados vetores, que representam os loadings. Esses vetores indicam quais variáveis foram as responsáveis pelo deslocamento e, ou, agrupamento de amostras.

\section{RESULTADOS E DISCUSSÃO}

\section{Chumbo}

Todos os perfis sob influência direta das atividades de mineração e metalurgia (solos 2 a 8) (Quadro 1 e Figura 1) apresentaram altos teores de $\mathrm{Pb}$ total (Quadro 5). Esses teores de $\mathrm{Pb}$ são superiores aos teores de intervenção residencial $\left(300 \mathrm{mg} \mathrm{kg}^{-1}\right)$ ou industrial (900 $\mathrm{mg} \mathrm{kg}^{-1}$ ) sugeridos pelo CONAMA (2009). Mesmo o solo 1, tomado como referência,

\section{Quadro 4. Extração sequencial para determinação das formas de $\mathrm{Pb}$ e $\mathrm{Zn}$ nas amostras dos solos}

\begin{tabular}{|c|c|c|c|}
\hline Fração & Extrator & Solo:Solução & Condições de Extração \\
\hline & \multicolumn{3}{|c|}{$\mathrm{g}: \mathrm{mL}$} \\
\hline Solúvel & $\mathrm{H}_{2} \mathrm{O}$ ultrapura (Mille-Q) & $2: 10$ & $\begin{array}{l}\text { Agitação por } 30 \text { min; centrifugação; } \\
\text { lavagem do resíduo. }\end{array}$ \\
\hline Trocável $^{(1)}$ & $\mathrm{Ca}\left(\mathrm{NO}_{3}\right)_{2} 0,5 \mathrm{~mol} \mathrm{~L}^{-1}$ & $2: 20$ & $\begin{array}{l}\text { Agitação por } 1 \mathrm{~h} \text {; centrifugação; } \\
\text { lavagem do resíduo. }\end{array}$ \\
\hline Carbonato $^{(2)}$ & Acetato de sódio $1,0 \mathrm{~mol} \mathrm{~L}^{-1}$ a pH 5 & $2: 20$ & $\begin{array}{l}\text { Agitação por } 5 \text { h; centrifugação; } \\
\text { lavagem do resíduo. }\end{array}$ \\
\hline Matéria Orgânica $^{(3)}$ & Hipoclorito de Sódio $0,7 \mathrm{~mol} \mathrm{~L}^{-1}$ & $2: 6$ & $\begin{array}{l}\text { Banho-maria a } 90^{\circ} \mathrm{C} \text { por } 30 \text { min; } \\
\text { centrifugação; lavagem do resíduo. }\end{array}$ \\
\hline $\begin{array}{l}\text { Óxidos de } \mathrm{Fe} \text { e } \mathrm{Al} \\
\operatorname{amorfos}^{(4)}\end{array}$ & $\begin{array}{l}\text { Oxalato de amônio } 0,2 \mathrm{~mol} \mathrm{~L}^{-1}+ \\
\text { ácido oxálico } 0,2 \mathrm{~mol} \mathrm{~L}^{-1}+\text { ácido } \\
\text { ascórbico } 0,1 \mathrm{~mol} \mathrm{~L}^{-1}\end{array}$ & $2: 40$ & $\begin{array}{l}\text { Agitação por } 4 \mathrm{~h} \text { no escuro; } \\
\text { centrifugação; lavagem do resíduo. }\end{array}$ \\
\hline $\begin{array}{l}\text { Óxidos de } \mathrm{Al} \text { cristalinos } \\
\text { e minerais } 1: 1 \text { e } 2: 1^{(5)}\end{array}$ & $\mathrm{NaOH} 1,25 \mathrm{~mol} \mathrm{~L}^{-1}$ & $2: 40$ & $\begin{array}{l}\text { Banho-maria a } 75^{\circ} \mathrm{C} \text { por } 1 \mathrm{~h} \text {; } \\
\text { centrifugação; lavagem do resíduo. }\end{array}$ \\
\hline Residual & $\mathrm{HNO}_{3}$ conc. $+\mathrm{HF}$ conc. $+\mathrm{H}_{2} \mathrm{O}_{2} 30 \%$ & $0,25: 8$ & $\begin{array}{l}\text { Digestão em forno de micro-ondas a } \\
200^{\circ} \mathrm{C} \text {; filtração. }\end{array}$ \\
\hline
\end{tabular}

(1) Adsorção não específica ou de esfera externa. ${ }^{(2)}$ Precipitado na forma de carbonatos. ${ }^{(3)}$ Ocluído ou adsorvido especificamente (esfera interna) nos coloides orgânicos. ${ }^{(4)}$ Ocluído ou adsorvido especificamente nos óxidos de Fe e Al amorfos. ${ }^{(5)}$ Ocluído ou adsorvido especificamente nos óxidos de Al cristalinos e minerais secundários filossilicatados (1:1 e 2:1). 
Quadro 5. Teores de $\mathrm{Pb}$ total e teores obtidos nas extrações sequenciais das amostras dos $\operatorname{solos}^{(1)}$

\begin{tabular}{|c|c|c|c|c|c|c|c|c|}
\hline Amostra & Pb total & Solúvel & Trocável & Carbonato & $\begin{array}{l}\text { Matéria } \\
\text { orgânica }\end{array}$ & $\begin{array}{c}\text { Óx. Fe e } \\
\text { Al amorfo }\end{array}$ & $\begin{array}{c}\text { Óx. Al } \\
\text { cristalino }\end{array}$ & Residual \\
\hline & \multicolumn{8}{|c|}{$\mathrm{mg} \mathrm{kg}^{-1}$} \\
\hline $1(0-10 \mathrm{~cm})$ & 426,3 & 0,3 & nd & 140,6 & $59,9(93)$ & $86,0(92)$ & $39,1(87)$ & 234,4 \\
\hline $1(10-20 \mathrm{~cm})$ & 87,4 & nd & 0,5 & 6,0 & $13,1(92)$ & 34,7 (91) & $19,8(88)$ & 25,2 \\
\hline $1(20-40 \mathrm{~cm})$ & 33,3 & 0,3 & nd & 1,4 & $0,5(90)$ & $26,0(90)$ & nd & 6,7 \\
\hline $2(0-10 \mathrm{~cm})$ & 3988,8 & 4,2 & 74,3 & 1330,5 & $947,9(98)$ & $525,5(98)$ & $565,9(92)$ & 745,4 \\
\hline $2(10-20 \mathrm{~cm})$ & 2995,4 & 2,7 & 9,8 & 761,8 & $1566,9(97)$ & $494,2(98)$ & 200,3 (93) & 1598,0 \\
\hline $2(20-40 \mathrm{~cm})$ & 3123,2 & 3,2 & 9,6 & 817,9 & $2135,1(98)$ & $596,4(99)$ & $3058,7(93)$ & 936,9 \\
\hline $3(0-10 \mathrm{~cm})$ & 15293,1 & 21,5 & 783,3 & 8234,2 & $4378,0(97)$ & $498,7(98)$ & $1925,3(94)$ & 4104,0 \\
\hline $3(10-20 \mathrm{~cm})$ & 17913,2 & 32,6 & 670,8 & 6430,7 & $4548,7(98)$ & $637,3(97)$ & $5537,7(93)$ & 4906,3 \\
\hline $3(20-40 \mathrm{~cm})$ & 19434,0 & 30,7 & 903,6 & 9692,8 & $3601,4(98)$ & $520,6(98)$ & $564,9(94)$ & 3599,8 \\
\hline $4(0-10 \mathrm{~cm})$ & 3892,7 & 1,6 & 2,9 & 959,8 & $1087,5(96)$ & 386,7 (95) & $651,5(90)$ & 868,4 \\
\hline $4(10-20 \mathrm{~cm})$ & 7227,9 & 0,2 & 2,7 & 1561,4 & $2021,2(97)$ & $494,1(96)$ & $1204,1(91)$ & 2495,9 \\
\hline $4(20-40 \mathrm{~cm})$ & 5021,2 & 0,3 & 2,2 & 976,8 & $104,1(96)$ & $544,3(97)$ & $1470,8(91)$ & 2512,4 \\
\hline $5(0-10 \mathrm{~cm})$ & 14530,1 & 6,1 & 6,0 & 1913,5 & $275,3(94)$ & 274,0 (95) & $1571,6(90)$ & 13428,4 \\
\hline $5(10-20 \mathrm{~cm})$ & 5806,8 & 1,2 & 3,3 & 2110,3 & $247,4(96)$ & $282,1(97)$ & $1713,4(92)$ & 1707,1 \\
\hline $5(20-40 \mathrm{~cm})$ & 10140,1 & 0,6 & 4,3 & 2287,8 & $359,5(97)$ & $234,2(97)$ & $2299,9(92)$ & 5298,2 \\
\hline $6(0-10 \mathrm{~cm})$ & 695,9 & 4,0 & 124,2 & 190,7 & $1,9(96)$ & $280,0(96)$ & $40,0(92)$ & 57,6 \\
\hline $6(10-20 \mathrm{~cm})$ & 240,9 & 0,6 & 12,8 & 65,2 & nd & $122,1(95)$ & nd & nd \\
\hline $6(20-40 \mathrm{~cm})$ & 105,4 & 0,1 & 3,2 & 19,8 & nd & $47,6(90)$ & nd & nd \\
\hline $7(0-10 \mathrm{~cm})$ & 2570,6 & 4,5 & 12,2 & 799,2 & 399,6 (99) & $344,8(98)$ & $351,1(96)$ & 1332,5 \\
\hline $7(10-20 \mathrm{~cm})$ & 1266,7 & 0,3 & 3,4 & 273,3 & 307,6 (100) & $231,7(100)$ & $147,1(98)$ & 598,4 \\
\hline $7(20-40 \mathrm{~cm})$ & 1270,6 & 0,4 & 0,5 & 237,2 & $336,8(100)$ & $310,4(100)$ & $171,9(98)$ & 552,4 \\
\hline $8(0-10 \mathrm{~cm})$ & 4796,3 & 1,9 & 9,2 & 3193,3 & $396,7(93)$ & $288,2(92)$ & $873,3(90)$ & 2219,2 \\
\hline $8(10-20 \mathrm{~cm})$ & 6062,1 & 1,5 & 6,8 & 2960,7 & $418,6(92)$ & $455,3(92)$ & $833,4(89)$ & 1628,4 \\
\hline $8(20-40 \mathrm{~cm})$ & 2807,4 & nd & 1,0 & 1153,3 & $430,5(93)$ & 259,7 (93) & $402,5(90)$ & 644,8 \\
\hline
\end{tabular}

(1) Extrações sequenciais: Solúvel $\left(\mathrm{H}_{2} \mathrm{O}\right)$, Trocável $\left(\mathrm{Ca}\left(\mathrm{NO}_{3}\right)_{2}\right)$, Carbonatos (Acetato de Sódio), Matéria Orgânica (Hipoclorito de Sódio), Óxidos de $\mathrm{Fe}$ e Al amorfos (Oxalato de Amônio), Óxidos de Al cristalinos e minerais secundários filossilicatados (1:1 e 2:1) $\left(\mathrm{NaOH} 1,25 \mathrm{~mol} \mathrm{~L}^{-1}\right)$, Residual $\left(\mathrm{HF} / \mathrm{HNO}_{3} / \mathrm{H}_{2} \mathrm{O}_{2}\right)$. Valores entre parênteses (em \%) podem ser usados para calcular os teores de $\mathrm{Pb}$ (subestimação), caso não houvesse o controle de perda de massa nas amostras nas extrações sequenciais (ver detalhes no texto). Os somatórios dos teores das extrações sequenciais não se aproximam dos teores totais devido ao controle de perda de massa das amostras: os teores de $\mathrm{Pb}$ das extrações sequenciais foram obtidos levando-se em conta a massa removida no tratamento anterior (os dados percentuais corrigidos de recuperação de $\mathrm{Pb}$ total nas extrações sequenciais são apresentados no quadro 6). nd = teores não detectados pelo método analítico (ICP-AES).

apresentou teor de $\mathrm{Pb}$ na camada de 0 a $10 \mathrm{~cm}$ superior a $180 \mathrm{mg} \mathrm{kg}^{-1}$, limite para intervenção agrícola, o que é coerente com a ocorrência de veios de galena $(\mathrm{PbS})$ nos materiais de origem da região (principalmente complexos granitoides e dolomitos e carbonatos com baixo grau metamórfico) (Oliveira et al., 2002).

Não se verificaram correlações entre os teores totais e teores das extrações sequenciais de $\mathrm{Pb}$ (Quadro 5) e os teores de carbono orgânico, $\mathrm{pH}$ e demais características químicas (Quadro 2). Yamashita (2004) trabalhou com amostras de solo da região do Vale do Ribeira, no município de Iporanga, e obteve o mesmo comportamento. Essa falta de relação causaefeito entre os teores de $\mathrm{Pb}$ e características químicas é atribuída à contaminação mais intensa de determinadas áreas e camadas de solos, ou seja, a poluição não foi homogênea na área.

No tocante à extração sequencial (Quadros 5 e 6), é importante destacar que neste trabalho tomou-se o cuidado de pesar as amostras antes e após cada etapa da extração, o que não é usual na maioria dos métodos propostos na literatura: usa-se apenas a massa inicial da amostra na primeira extração para calcular o teor de metais pesados em todas as etapas subsequentes, sem considerar que, mesmo sendo extrações parciais, verifica-se remoção de parte da fase mineral e orgânica em questão. Os valores percentuais entre parênteses no quadro 5 significam que, caso não tivesse sido tomado esse cuidado com a pesagem, os teores nas extrações sequenciais que promovem redução de massa estariam, em sua maioria, subestimados, chegando-se em até $13 \%$ de erro em apenas uma extração, como é o caso do tratamento com $\mathrm{NaOH}$ $1,25 \mathrm{~mol} \mathrm{~L}^{-1}$ na amostra $1(0-10 \mathrm{~cm})(39,1 \times 0,87=$ $\left.34,0 \mathrm{mg} \mathrm{kg}^{-1}\right)$. Esse efeito é ainda mais expressivo quando se considera o erro acumulado nas extrações sequenciais. Dessa forma, sugere-se que, ao longo da utilização do método, sejam acompanhadas as variações de massa das amostras para dar maior confiabilidade aos resultados, expressando-se os reais teores dos metais em cada fração do solo. Outros autores também tomaram o mesmo cuidado de controle de massa de amostras de argila em tratamentos sequenciais para $\mathrm{Pb}$ (Wowk \& Melo, 2005) e para K e Mg (Melo et al., 2002, 2003). 
Somando os teores solúveis e trocáveis (formas mais disponíveis), observa-se que os solos 3 e $6(0-10 \mathrm{~cm})$ apresentam maiores riscos ambientais. A adsorção não específica de $\mathrm{Pb}$ no solo 3 (teor trocável máximo de $903,6 \mathrm{mg} \mathrm{kg}^{-1}$ ) foi favorecida pela intensa contaminação desse ponto (elevados teores totais do metal Quadro 5), presença de esmectita (Quadro 3) e alta CTC a pH 7,0 (Quadro 2). Os materiais particulados resultantes das chaminés depositaram neste local grande quantidade de $\mathrm{Pb}$, pois as partículas emitidas tendem a cair próximas à fonte de emissão (Quadro 1). As atividades de mineração e de processamento de minérios sulfetados de $\mathrm{Pb}$ produzem, além de rejeitos e escória de forno, emissões gasosas contendo $\mathrm{SO}_{2} \mathrm{CO}$ e poeiras contendo partículas com 20 a $65 \mathrm{dag} \mathrm{kg}^{-1} \mathrm{de}$ $\mathrm{Pb}$ (Burgess, 1995). Barros et al. (2010a) trabalharam com parte dos solos do presente estudo e interpretaram o maior número de bactérias esporuláveis no solo 3 como mecanismo de resistência desses organismos aos elevados teores de $\mathrm{Pb}$ biodisponíveis. Como consequência, a alta fitotoxidez por $\mathrm{Pb}$ nesse solo limitou severamente o crescimento de plantas de girassol, aveia-preta e grama-batatais em estudo de fitorremediação (Andrade et al., 2009). Dessa forma, uma das técnicas que podem ser indicadas para remediação desse ponto é a remoção direta do solo.
Com relação às formas mais estáveis de $\mathrm{Pb}$ (precipitado - carbonato; adsorvidos por complexos de esfera interna - matéria orgânica, óxidos de $\mathrm{Fe}$ e $\mathrm{Al}$ de baixa cristalinidade, óxidos de $\mathrm{Al}$ cristalinos e minerais filossilicatados secundários (1:1 e 2:1); e na estrutura de minerais - residual), tomando os valores médios (Quadro 6), observou-se maior associação aos carbonatos, seguida de fração residual e óxidos de $\mathrm{Fe}$ e $\mathrm{Al}$ amorfos. A ocorrência de rochas calcárias na região e os elevados valores de $\mathrm{pH}$ dos solos (Quadro 2) favorecem a precipitação do $\mathrm{Pb}$ na forma de carbonatos (Sposito et al., 1982; Amaral Sobrinho et al., 2009). Corsi \& Landim (2002) obtiveram em sedimentos da região do Vale do Ribeira contribuições de carbonatos (em média, 22,36 \%) semelhantes ás do presente trabalho, porém a maior parte do $\mathrm{Pb}$ estava associada aos óxidos de $\mathrm{Fe}$ e $\mathrm{Mn}$. A alta percentagem de $\mathrm{Pb}$ carbonato (Quadro 6), aliada aos altos teores extraídos pelo acetato de sódio (Quadro 5), torna esse ambiente com grandes riscos ambientais sob futuras condições de acidificação do solo. Contudo, deve-se considerar a relativa falta de seletividade de alguns extratores nas análises sequenciais (Amaral Sobrinho et al., 2009).

No solo 5, devido à grande ocorrência de resíduos da metalurgia incorporados e, principalmente, na

Quadro 6. Contribuição percentual das extrações sequenciais em relação ao $\mathrm{Pb}$ total dos solos, levando-se em conta a perda de massa da amostra em cada extração ${ }^{(1)}$

\begin{tabular}{|c|c|c|c|c|c|c|c|c|}
\hline Amostra & $\mathrm{Pb}$ total & Solúvel & Trocável & Carbonato & $\begin{array}{c}\text { Matéria } \\
\text { orgânica }\end{array}$ & $\begin{array}{c}\text { Óx. Fe e } \\
\text { Al amorfo }\end{array}$ & $\begin{array}{c}\text { Óx. Al } \\
\text { cristalino }\end{array}$ & Residual \\
\hline & $\mathrm{mg} \mathrm{kg}^{-1}$ & & & & $\%$ & & & \\
\hline $1(0-10 \mathrm{~cm})$ & 426,3 & 0,1 & 0,0 & 26,7 & 11,1 & 15,9 & 6,9 & 39,4 \\
\hline $1(10-20 \mathrm{~cm})$ & 87,4 & 0,0 & 0,5 & 6,4 & 13,6 & 36,0 & 19,6 & 23,9 \\
\hline $1(20-40 \mathrm{~cm})$ & 33,3 & 0,8 & 0,0 & 4,2 & 1,3 & 75,9 & 0,0 & 17,8 \\
\hline $2(0-10 \mathrm{~cm})$ & 3988,8 & 0,1 & 1,9 & 33,1 & 22,9 & 12,7 & 13,2 & 16,2 \\
\hline $2(10-20 \mathrm{~cm})$ & 2995,4 & 0,1 & 0,2 & 17,3 & 34,7 & 10,9 & 4,2 & 32,7 \\
\hline $2(20-40 \mathrm{~cm})$ & 3123,2 & 0,0 & 0,1 & 11,3 & 28,9 & 8,0 & 39,7 & 11,9 \\
\hline $3(0-10 \mathrm{~cm})$ & 15293,1 & 0,1 & 4,1 & 43,4 & 22,2 & 2,5 & 9,2 & 18,5 \\
\hline $3(10-20 \mathrm{~cm})$ & 17913,2 & 0,2 & 3,1 & 30,0 & 20,5 & 2,9 & 23,5 & 19,8 \\
\hline $3(20-40 \mathrm{~cm})$ & 19434,0 & 0,2 & 5,0 & 53,2 & 19,0 & 2,8 & 2,8 & 17,1 \\
\hline $4(0-10 \mathrm{~cm})$ & 3892,7 & 0,0 & 0,1 & 25,5 & 27,7 & 9,8 & 16,0 & 20,9 \\
\hline $4(10-20 \mathrm{~cm})$ & 7227,9 & 0,0 & 0,0 & 21,4 & 26,4 & 6,4 & 15,0 & 30,8 \\
\hline $4(20-40 \mathrm{~cm})$ & 5021,2 & 0,0 & 0,0 & 19,5 & 1,9 & 9,8 & 25,6 & 43,2 \\
\hline $5(0-10 \mathrm{~cm})$ & 14530,1 & 0,0 & 0,0 & 12,5 & 1,7 & 1,7 & 9,0 & 75,2 \\
\hline $5(10-20 \mathrm{~cm})$ & 5806,8 & 0,0 & 0,1 & 37,9 & 4,1 & 4,6 & 27,1 & 26,2 \\
\hline $5(20-40 \mathrm{~cm})$ & 10140,1 & 0,0 & 0,1 & 24,3 & 3,5 & 2,2 & 21,5 & 48,5 \\
\hline $6(0-10 \mathrm{~cm})$ & 695,9 & 0,6 & 18,0 & 27,7 & 0,3 & 40,0 & 5,6 & 8,0 \\
\hline $6(10-20 \mathrm{~cm})$ & 240,9 & 0,3 & 6,4 & 32,6 & 0,0 & 60,7 & 0,0 & 0,0 \\
\hline $6(20-40 \mathrm{~cm})$ & 105,4 & 0,1 & 4,6 & 28,0 & 0,0 & 67,3 & 0,0 & 0,0 \\
\hline $7(0-10 \mathrm{~cm})$ & 2570,6 & 0,2 & 0,4 & 26,8 & 12,6 & 10,8 & 10,6 & 38,8 \\
\hline $7(10-20 \mathrm{~cm})$ & 1266,7 & 0,0 & 0,2 & 18,7 & 20,2 & 15,2 & 9,2 & 36,5 \\
\hline $7(20-40 \mathrm{~cm})$ & 1270,6 & 0,0 & 0,0 & 15,7 & 21,6 & 19,9 & 10,5 & 32,2 \\
\hline $8(0-10 \mathrm{~cm})$ & 4796,3 & 0,0 & 0,1 & 48,6 & 5,6 & 4,0 & 11,9 & 29,7 \\
\hline $8(10-20 \mathrm{~cm})$ & 6062,1 & 0,0 & 0,1 & 49,8 & 6,5 & 7,0 & 12,5 & 24,0 \\
\hline $8(20-40 \mathrm{~cm})$ & 2807,4 & 0,0 & 0,0 & 42,3 & 14,7 & 8,9 & 13,3 & 20,8 \\
\hline Média & & 0,1 & 1,9 & 27,4 & 13,4 & 18,2 & 12,8 & 26,3 \\
\hline
\end{tabular}

(1) Extrações sequenciais: Solúvel $\left(\mathrm{H}_{2} \mathrm{O}\right)$, Trocável $\left(\mathrm{Ca}\left(\mathrm{NO}_{3}\right)_{2}\right)$, Carbonatos (Acetato de Sódio), Matéria Orgânica (Hipoclorito de Sódio), Óxidos de $\mathrm{Fe}$ e $\mathrm{Al}$ amorfos (Oxalato de Amônio), Óxidos de Al cristalinos e minerais secundários filossilicatados (1:1 e 2:1) ( $\mathrm{NaOH} 1,25 \mathrm{~mol} \mathrm{~L}^{-1}$ ), Residual ( $\left.\mathrm{HF} / \mathrm{HNO}_{3} / \mathrm{H}_{2} \mathrm{O}_{2}\right)$. Média: contribuição média das extrações sequenciais no $\mathrm{Pb}$ total. 
superfície do solo (Quadro 1), 75,2\% do Pb total está distribuído na fração residual na camada de 0 a $10 \mathrm{~cm}$, o que indica baixa solubilidade desses resíduos. Esses resultados sugerem a remoção imediata dos resíduos depositados na área como prática de remediação. Em estudo de cinética de liberação de $\mathrm{Pb}$, Buschle et al. (2010) observaram elevado tamponamento do metal no solo 5, o que indica contaminação contínua ao longo do tempo.

No solo 6 (adição de $\mathrm{Pb}$ particulado via chaminé da fábrica - Quadro 1), o efeito do material amorfo extraído com oxalato de amônio na retenção de $\mathrm{Pb}$ foi intensificado (Quadro 6), devido aos seus baixos teores de matéria orgânica e argila (Quadro 2). Já no solo 8 - área entre a fábrica e o rio Ribeira (Figura 1), onde a principal fonte de $\mathrm{Pb}$ é a água pluvial que lava a fábrica e deságua no rio - as formas solúveis do metal precipitaram com os carbonatos (valores de $\mathrm{pH}$ superiores a 8,2 - Quadro 2), e a extração com acetato de sódio foi responsável por mais de $40 \%$ do $\mathrm{Pb}$ total (Quadro 6)

A ausência de correlações significativas entre os minerais de baixa e alta cristalinidade da fração argila (Quadro 3) e as respectivas frações na extração sequencial (Quadros 5 e 6) é atribuída à contaminação heterogênea da área, a remoções apenas parciais dos componentes (extrações com baixa energia) e às diferenças qualitativas dos minerais: área superficial específica, cristalinidade, etc.

Os resultados das percentagens do $\mathrm{Pb}$ total em cada fração foram ordenados por meio da análise por componentes principais (PCA) (Figura 2), em que os componentes principais 1 e 2 (PCA-1 e PCA-2) explicaram 72,48 \%: a PCA-1 explicou 50,97\%, e a PCA-2, 21,51 \% da variabilidade total dos dados. Os escores do componente principal 1 (PCA-1) correlacionaram-se positivamente com a $\% \mathrm{~Pb}$ Sol $(\mathrm{r}$ $=0,78), \% \mathrm{~Pb}$ Óx. Fe e $\mathrm{Al}(\mathrm{r}=0,77)$ e $\% \mathrm{~Pb}$ Troc $(\mathrm{r}=$ $0,70)$; negativamente, com a $\% \mathrm{~Pb} \mathrm{O} x$. $\mathrm{Al}(\mathrm{r}=$ $0,86), \% \mathrm{~Pb} \operatorname{Res}(r=-0,82)$ e $\% \mathrm{~Pb} \mathrm{MO}(\mathrm{r}=-0,71)$. A componente principal 2 correlacionou-se negativamente com a \%Pb Carb $(r=-0,94)$.

Por essa análise, observou-se adequado agrupamento das amostras do solo 6 (Figura 2). O isolamento dessas amostras no quadrante inferior direito no gráfico da PCA foi fortemente influenciado pelas maiores percentagem de $\mathrm{Pb}$ trocável - observar a direção da seta da variável \% Pb trocável e os dados do quadro 6. A amostra mais profunda do ponto 1 (20-40 cm) distanciou-se graficamente das demais por possuir característica diferenciada: maior \% de $\mathrm{Pb}$ ligado aos óxidos de $\mathrm{Fe}$ e $\mathrm{Al}$ de baixa cristalinidade (Quadro 6). As altas \% $\mathrm{Pb}$ carbonato direcionaram as amostras dos solos 3 e 8 para a parte inferior do gráfico de PCA. O posicionamento das amostras do solo 8, em relação ao solo 3 , mais próximo da linhalimite dos quadrantes inferior e superior, foi influenciado por seus menores valores de $\% \mathrm{~Pb}$ trocável (posicionamento em sentido oposto ao vetor dessa variável) (Figura 2). A amostra 5A posicionouse em outro quadrante em relação às demais amostras desse solo, por apresentar maiores valores de $\% \mathrm{~Pb}$ residual. Conforme já destacado, isso indica

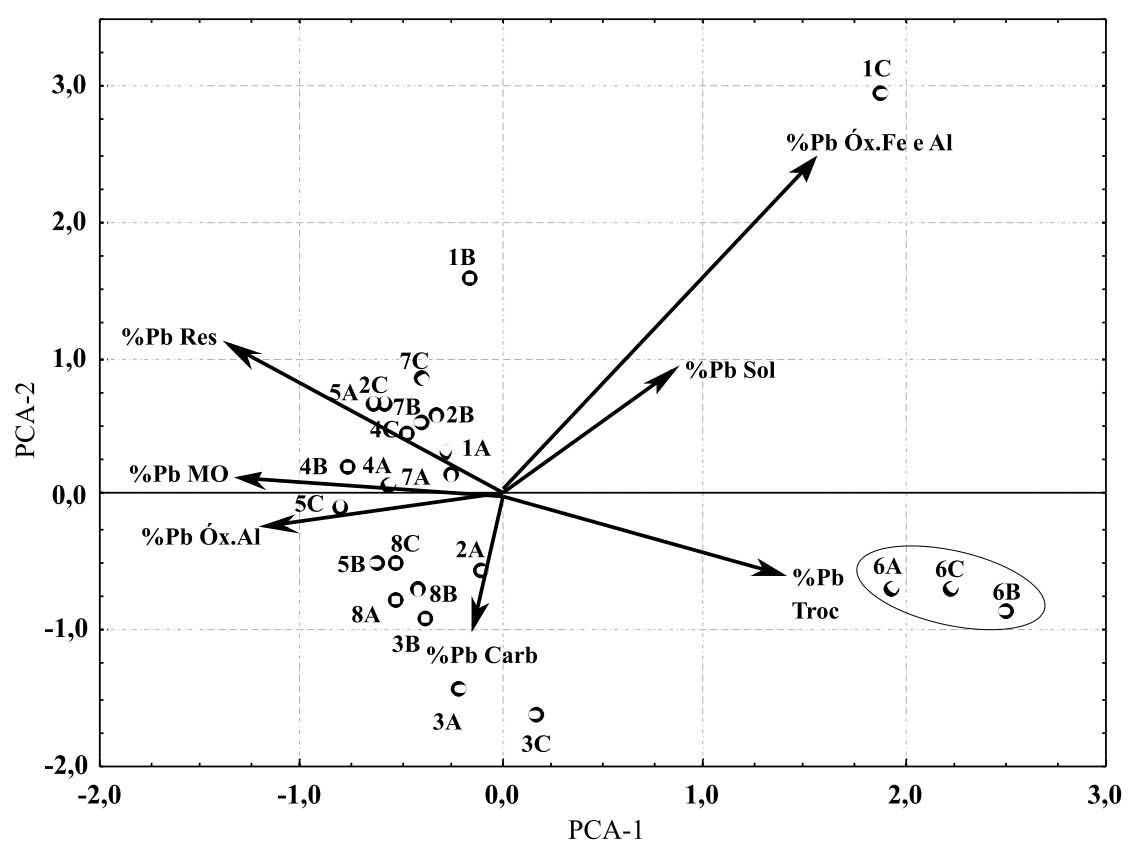

Figura 2. Gráfico dos componentes principais (PCA-1 e PCA-2) das amostras e da distribuição percentual do Pb total em cada fração da análise sequencial: Sol.: solúvel; Troc.: trocável; Carb: carbonatos; MO: matéria orgânica; Óx. Fe e Al: óxidos de F e Al amorfos; Óx. Al: óxidos de Al cristalinos e minerais secundários filossilicatados (1:1 e 2:1); Res: residual. NOTA: O número representa o ponto de coleta, e a letra, a profundidade. $(A=0$ a $10 \mathrm{~cm} ; B=10$ a $20 \mathrm{~cm} ; C=20$ a $40 \mathrm{~cm})$. 
tamponamento a longo prazo de $\mathrm{Pb}$ na camada superficial do solo 5 .

\section{Zinco}

Os maiores teores totais de $\mathrm{Zn}$ foram observados na camada mais superficial dos solos $(0-10 \mathrm{~cm})$ (Quadro 7). Foi possível observar que existem dois grupos distintos de amostras: os solos 3, 5 e 8 são os mais críticos, pois apresentaram teores de $\mathrm{Zn}$ maiores que $400 \mathrm{mg} \mathrm{kg}^{-1}$ e concentração acima do limite tóxico às plantas (Mattiazzo et al., 2001), enquadrando-se, segundo CONAMA (2009), em intervenção para uso agrícola. Os demais solos encontram-se dentro do limite de qualidade de solo estabelecidos por esse órgão ambiental.

Guimarães (2007) correlacionou os teores totais de $\mathrm{Pb}$ e Zn encontrados nos rejeitos de mineração da Mina do Rocha (Vale do Ribeira) e obteve correlações expressivas entre esses metais ( $r$ entre teores de $\mathrm{Pb}$ e $\mathrm{Zn}=0,90 \mathrm{p}<0,05)$. Tomando os teores das extrações sequenciais de $\mathrm{Pb}$ e $\mathrm{Zn}$ (Quadros 5 e 7), observaramse correlações significativas entre as frações trocáveis e residuais para esses metais $(r=0,99 p<0,01$ e 0,84 $\mathrm{p}<0,05$, respectivamente).
O solo 1 teve a maior contribuição da fração solúvel em relação ao Zn total do solo (Quadro 8), porém esses valores não representam potencial poluidor, visto que o teor total de $\mathrm{Zn}$ nesse ponto foi baixo.

Em relação ao $\mathrm{Pb}$, verificou-se a tendência de os solos mais contaminados apresentarem $\mathrm{Zn}$ em formas mais insolúveis, aumentando a participação da fração residual nos teores totais (Quadros 7 e 8). Gomes et al. (1997) atribuem as elevadas concentrações de metais pesados associados à fração residual, frequentemente, à baixa capacidade de solubilização da fração óxidos de Fe cristalinos pela solução extratora. Quando o pH do solo é elevado (próximo ou acima da neutralidade), o Zn é mais adsorvido pelos óxidos e hidróxidos de Fe, de $\mathrm{Al}$ e de Mn (Stahl \& James, 1991; Zhang et al. (1997). Sims (1986) trabalhou com solo arenoso com baixo teor de matéria orgânica e verificou que, numa variação de pH de 4,8 a 7,7, o Zn ligado aos óxidos de Fe amorfos aumentou de 4 para $26 \%$, enquanto o Zn trocável diminuiu de 42 para $1 \%$, respectivamente. A pequena contribuição do $\mathrm{Zn}$ presente na fração dos óxidos de $\mathrm{Al}$ cristalinos e minerais 1:1 e 2:1 (Quadro 8), possivelmente, deveu-se à remoção prévia dos óxidos de $\mathrm{Fe}$ e $\mathrm{Al}$ mais reativos com oxalato de amônio.

Quadro 7. Teores de Zn total e teores obtidos nas extrações sequenciais das amostras dos solos ${ }^{(1)}$

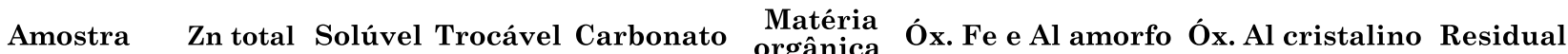

\begin{tabular}{|c|c|c|c|c|c|c|c|c|}
\hline & & & & & & & & \\
\hline $1(0-10 \mathrm{~cm})$ & 70,0 & 1,2 & nd & 24,4 & 7,7 & 37,4 & nd & 57,0 \\
\hline $1(10-20 \mathrm{~cm})$ & 16,6 & 1,3 & nd & nd & nd & nd & nd & 17,8 \\
\hline $1(20-40 \mathrm{~cm})$ & 8,8 & 1,5 & nd & 23,3 & 0,9 & 9,1 & nd & 22,0 \\
\hline $2(0-10 \mathrm{~cm})$ & 135,6 & 1,8 & 16,2 & 0,0 & nd & 22,2 & nd & 66,5 \\
\hline $2(10-20 \mathrm{~cm})$ & 92,4 & 0,4 & 4,7 & 0,0 & nd & 2,1 & nd & 170,0 \\
\hline $2(20-40 \mathrm{~cm})$ & 83,2 & 0,4 & 10,6 & 3,0 & 5,2 & 2,9 & 38,1 & 88,4 \\
\hline $3(0-10 \mathrm{~cm})$ & 1361,4 & 4,3 & 206,9 & 191,4 & 177,9 & 361,1 & 32,7 & 517,5 \\
\hline $3(10-20 \mathrm{~cm})$ & 1618,6 & 4,5 & 199,9 & 198,0 & 189,0 & 527,6 & 48,5 & 524,7 \\
\hline $3(20-40 \mathrm{~cm})$ & 1481,4 & 4,9 & 237,3 & 211,9 & 156,6 & 458,8 & nd & 510,1 \\
\hline $4(0-10 \mathrm{~cm})$ & 142,7 & 0,3 & nd & 8,5 & 26,2 & 64,0 & nd & 93,0 \\
\hline $4(10-20 \mathrm{~cm})$ & 45,8 & nd & nd & 4,6 & 17,2 & 18,9 & nd & 58,1 \\
\hline $4(20-40 \mathrm{~cm})$ & 50,0 & nd & 2,2 & 46,7 & nd & 89,4 & nd & 68,2 \\
\hline $5(0-10 \mathrm{~cm})$ & 19719,7 & 9,3 & 16,4 & 2354,1 & 316,6 & 622,8 & 815,1 & 20257,8 \\
\hline $5(10-20 \mathrm{~cm})$ & 1471,1 & 0,9 & nd & 67,8 & nd & 221,6 & 42,6 & 814,2 \\
\hline $5(20-40 \mathrm{~cm})$ & 1143,4 & 0,3 & nd & 51,9 & nd & 486,1 & 30,1 & 729,1 \\
\hline $6(0-10 \mathrm{~cm})$ & 83,2 & 0,2 & 40,2 & 64,8 & nd & 55,0 & nd & nd \\
\hline $6(10-20 \mathrm{~cm})$ & 35,4 & 0,3 & 12,3 & 21,0 & nd & 86,8 & nd & nd \\
\hline $6(20-40 \mathrm{~cm})$ & 16,9 & 0,2 & 0,2 & nd & nd & 28,2 & nd & nd \\
\hline $7(0-10 \mathrm{~cm})$ & 234,6 & 1,6 & nd & 25,9 & 10,9 & 99,3 & nd & 160,8 \\
\hline $7(10-20 \mathrm{~cm})$ & 44,1 & 0,1 & nd & nd & nd & 0,7 & nd & 48,4 \\
\hline $7(20-40 \mathrm{~cm})$ & 60,3 & 0,3 & nd & 9,1 & 15,0 & 27,3 & nd & 54,7 \\
\hline $8(0-10 \mathrm{~cm})$ & 3778,2 & 0,8 & 5,1 & 538,0 & 78,8 & 694,7 & 36,6 & 5961,9 \\
\hline $8(10-20 \mathrm{~cm})$ & 3118,9 & 0,6 & 8,7 & 385,0 & 75,4 & 817,7 & 39,4 & 2024,1 \\
\hline $8(20-40 \mathrm{~cm})$ & 1926,0 & 0,1 & nd & 192,5 & 102,5 & 374,8 & 9,5 & 1345,1 \\
\hline
\end{tabular}

(1) Extrações sequenciais: Solúvel $\left(\mathrm{H}_{2} \mathrm{O}\right)$, Trocável $\left(\mathrm{Ca}\left(\mathrm{NO}_{3}\right)_{2}\right)$, Carbonatos (Acetato de Sódio), Matéria Orgânica (Hipoclorito de Sódio), Óxidos de Fe e Al amorfos (Oxalato de Amônio), Óxidos de Al cristalinos e minerais secundários filossilicatados (1:1 e 2:1) $\left(\mathrm{NaOH} 1,25 \mathrm{~mol} \mathrm{~L}^{-1}\right)$, Residual $\left(\mathrm{HF} / \mathrm{HNO}_{3} / \mathrm{H}_{2} \mathrm{O}_{2}\right) . \mathrm{O}$ somatório dos teores das extrações sequenciais não se aproximam dos teores totais devido ao controle de perda de massa das amostras: os teores de Zn das extrações sequenciais foram obtidos levando-se em conta a massa removida no tratamento anterior (os dados percentuais corrigidos de recuperação de $\mathrm{Pb}$ total nas extrações sequenciais são apresentados no quadro 8). nd = teores não detectados pela técnica de dosagem (ICP-AES). 
Quadro 8. Contribuição percentual das extrações sequenciais em relação ao Zn total dos solos, levando-se em conta a perda de massa da amostra em cada extração(1)

\begin{tabular}{|c|c|c|c|c|c|c|c|c|}
\hline Amostra & Zn total & Solúvel & Trocável & Carbonato & $\begin{array}{c}\text { Matéria } \\
\text { orgânica }\end{array}$ & $\begin{array}{c}\text { Óx. Fe e } \\
\text { Al amorfo }\end{array}$ & $\begin{array}{c}\text { Óx. Al } \\
\text { cristalino }\end{array}$ & Residual \\
\hline & $\mathrm{mg} \mathrm{kg}^{-1}$ & & & & $\%$ & & & \\
\hline $1(0-10 \mathrm{~cm})$ & 70,0 & 1,0 & 0,0 & 20,3 & 6,3 & 30,4 & 0,0 & 42,1 \\
\hline $1(10-20 \mathrm{~cm})$ & 16,6 & 7,7 & 0,0 & 0,0 & 0,0 & 0,0 & 0,0 & 38,2 \\
\hline $1(20-40 \mathrm{~cm})$ & 8,8 & 2,7 & 0,0 & 42,8 & 1,7 & 16,4 & 0,0 & 36,4 \\
\hline $2(0-10 \mathrm{~cm})$ & 135,6 & 1,8 & 16,6 & 0,0 & 0,0 & 22,1 & 0,0 & 59,5 \\
\hline $2(10-20 \mathrm{~cm})$ & 92,4 & 0,3 & 2,9 & 0,0 & 0,0 & 1,3 & 0,0 & 95,5 \\
\hline $2(20-40 \mathrm{~cm})$ & 83,2 & 0,3 & 7,7 & 2,2 & 3,7 & 2,0 & 25,8 & 58,4 \\
\hline $3(0-10 \mathrm{~cm})$ & 1361,4 & 0,3 & 14,9 & 13,8 & 12,3 & 24,8 & 2,1 & 31,8 \\
\hline $3(10-20 \mathrm{~cm})$ & 1618,6 & 0,3 & 12,6 & 12,5 & 11,5 & 31,8 & 2,8 & 28,6 \\
\hline $3(20-40 \mathrm{~cm})$ & 1481,4 & 0,3 & 15,9 & 14,2 & 10,1 & 29,7 & 0,0 & 29,7 \\
\hline $4(0-10 \mathrm{~cm})$ & 142,7 & 0,2 & 0,0 & 4,8 & 14,0 & 34,1 & 0,0 & 47,0 \\
\hline $4(10-20 \mathrm{~cm})$ & 45,8 & 0,0 & 0,0 & 5,1 & 17,9 & 19,7 & 0,0 & 57,3 \\
\hline $4(20-40 \mathrm{~cm})$ & 50,0 & 0,0 & 1,2 & 24,9 & 0,0 & 42,7 & 0,0 & 31,3 \\
\hline $5(0-10 \mathrm{~cm})$ & 19719,7 & 0,0 & 0,1 & 11,0 & 1,4 & 2,7 & 3,3 & 81,4 \\
\hline $5(10-20 \mathrm{~cm})$ & 1471,1 & 0,1 & 0,0 & 6,7 & 0,0 & 20,2 & 3,7 & 69,3 \\
\hline $5(20-40 \mathrm{~cm})$ & 1143,4 & 0,0 & 0,0 & 4,5 & 0,0 & 38,3 & 2,3 & 54,9 \\
\hline $6(0-10 \mathrm{~cm})$ & 83,2 & 0,0 & 40,1 & 35,3 & 0,0 & 24,6 & 0,0 & 0,0 \\
\hline $6(10-20 \mathrm{~cm})$ & 35,4 & 0,0 & 16,3 & 25,0 & 0,0 & 58,7 & 0,0 & 0,0 \\
\hline $6(20-40 \mathrm{~cm})$ & 16,9 & 0,0 & 1,2 & 0,0 & 0,0 & 98,8 & 0,0 & 0,0 \\
\hline $7(0-10 \mathrm{~cm})$ & 234,6 & 0,6 & 0,0 & 9,6 & 3,8 & 34,5 & 0,0 & 51,6 \\
\hline $7(10-20 \mathrm{~cm})$ & 44,1 & 0,3 & 0,0 & 0,0 & 0,0 & 1,6 & 0,0 & 98,1 \\
\hline $7(20-40 \mathrm{~cm})$ & 60,3 & 0,3 & 0,0 & 9,2 & 14,8 & 26,8 & 0,0 & 48,9 \\
\hline $8(0-10 \mathrm{~cm})$ & 3778,2 & 0,0 & 0,1 & 8,2 & 1,1 & 9,8 & 0,5 & 80,3 \\
\hline $8(10-20 \mathrm{~cm})$ & 3118,9 & 0,0 & 0,3 & 12,8 & 2,3 & 24,8 & 1,2 & 58,7 \\
\hline $8(20-40 \mathrm{~cm})$ & 1926,0 & 0,0 & 0,0 & 10,5 & 5,2 & 19,1 & 0,5 & 64,7 \\
\hline Média & & 0,7 & 3,0 & 8,9 & 4,4 & 18,0 & 1,8 & 50,1 \\
\hline
\end{tabular}

(1) Extrações sequenciais: Solúvel $\left(\mathrm{H}_{2} \mathrm{O}\right)$, Trocável $\left(\mathrm{Ca}\left(\mathrm{NO}_{3}\right)_{2}\right)$, Carbonatos (Acetato de Sódio), Matéria Orgânica (Hipoclorito de Sódio), Óxidos de $\mathrm{Fe}$ e $\mathrm{Al}$ amorfos (Oxalato de Amônio), Óxidos de Al cristalinos e minerais secundários filossilicatados (1:1 e 2:1) $\left(\mathrm{NaOH} 1,25 \mathrm{~mol} \mathrm{~L}^{-1}\right)$, Residual $\left(\mathrm{HF} / \mathrm{HNO}_{3} / \mathrm{H}_{2} \mathrm{O}_{2}\right.$ ).

Os componentes principais 1 e 2 (PCA-1 e PCA-2) explicaram 55,83\%: a PCA-1 explicou 37,70 \%, e a PCA-2, 18,13\% da variabilidade total dos dados. Para o Zn, não foi possível bom agrupamento dos dados (Figura 3). O ponto $1(10-20 \mathrm{~cm})$ ficou visualmente mais deslocado, em razão da considerável contribuição

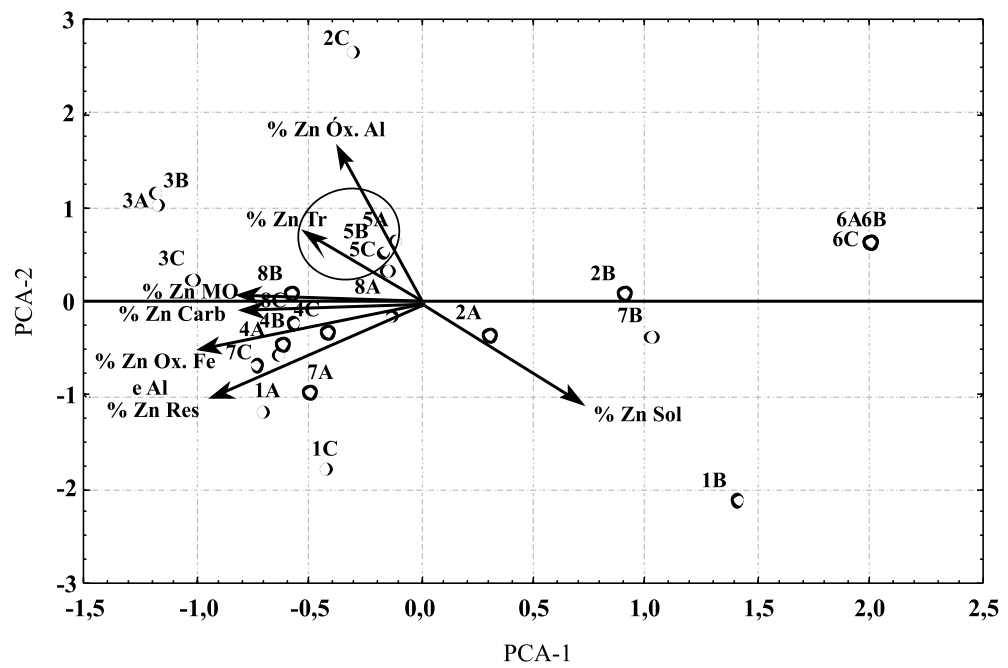

Figura 3. Gráfico dos componentes principais (PCA-1 e PCA-2) das amostras e da distribuição percentual do Zn total em cada fração da análise sequencial: Sol.: solúvel; Troc.: trocável; Carb: carbonatos; MO: matéria orgânica; Óx. Fe e Al: óxidos de F e Al amorfos; Óx. Al: óxidos de Al cristalinos e minerais secundários filossilicatados (1:1 e 2:1); Res: residual. NOTA: O número representa o ponto de coleta, e a letra, a profundidade. $(A=0$ a $10 \mathrm{~cm} ; B=10$ a $20 \mathrm{~cm} ; C=20$ a $40 \mathrm{~cm})$. 
da fração solúvel (Figura 3 e Quadro 8). Todas as amostras do ponto 5 ficaram próximas umas das outras, em função da similaridade da \% Zn óxidos de $\mathrm{Al}$ cristalinos e minerais 1:1 e 2:1.

\section{CONCLUSÕES}

1. A contaminação do solo argiloso (solo 3) próximo à fábrica por materiais particulados das chaminés mostrou-se mais prejudicial ao ambiente em virtude dos maiores teores totais e de formas de maior disponibilidade (solução do solo mais trocável).

2. Tomando os valores médios, houve maior associação do $\mathrm{Pb}$ aos carbonatos, seguida da fração residual e de óxidos de $\mathrm{Fe}$ e $\mathrm{Al}$ de baixa cristalinidade. $\mathrm{O} \mathrm{Zn}$ apresentou preferência por formas mais insolúveis, aumentando a participação da fração residual nos teores totais.

3. A análise por componentes principais foi sensível às diferentes formas de $\mathrm{Pb}$ nos solos e promoveu o agrupamento das amostras, principalmente, em função das formas trocáveis e associadas aos óxidos de $\mathrm{Fe}$ e de $\mathrm{Al}$ e aos carbonatos.

4. É necessário realizar a etapa de pesagem das amostras antes e após cada etapa da extração sequencial para evitar erros e, assim, dar maior confiabilidade aos resultados, expressando-se os reais teores dos metais em cada fração.

\section{LITERATURA CITADA}

AMARAL SOBRINHO, N.M.B.; VELLOSO, A.C.X.; COSTA, L.M. \& OLIVEIRA, C. Formas químicas de zinco e sua absorção por plantas de milho cultivadas em solo tratado com resíduo siderúrgico. R. Bras. Ci. Solo, 18:313-320, 1994.

AMARAL SOBRINHO, N.M.B.; BARRA, C.M. \& LÃ, O.R Química dos metais pesados nos solos. In: MELO, V.F. \& ALLEONI, L.R. (ed.). Química e mineralogia do solo: Aplicações. Viçosa, MG, Sociedade Brasileira de Ciência do Solo, 2009. Parte II. v.2. p.249-312.

ANDRADE, M. Teores de metais pesados em águas e em sedimentos de área de mineração e processamento de $\mathrm{Pb}$, no município de Adrianópolis-PR. Curitiba, Universidade Federal do Paraná, 2009. 114p. (Dissertação de Mestrado)

ANDRADE, M.G.; MELO, V.F.; GABARDO, J.; SOUZA, L.C.P \& REISSMANN, C.B. Metais pesados em solos de área de mineração e metalurgia de chumbo: I - Fitoextração. R. Bras. Ci. Solo, 33:1879-1888, 2009

BARROS, Y.J.; MELO, V.F.; DIONÍSIO, J.A.; CARON, L.; OLIVEIRA, E.B.; AZEVEDO, J.C.R. \& SOUZA, L.C.P. Indicadores de qualidade de solos de área de mineração e metalurgia de chumbo: I- microbiologia. R. Bras. Ci. Solo, 34:1397-1411, 2010a.
BARROS, Y.J.; MELO, V.F.; SAUTTER, K.D.; BUSCHLE, B.; OLIVEIRA, E.B.; AZEVEDO, J.C.R. \& SOUZA, L.C.P. Indicadores de qualidade de solos de área de mineração e metalurgia de chumbo: II - mesofauna e plantas. R. Bras. Ci. Solo, 34:1413-1426-2010b.

BOSSO, S.T. \& ENZWEILER, J. Ensaios para determinar a (Bio) disponibilidade de chumbo em solos contaminados: revisão. Quim. Nova, 31:394-400, 2008.

BURGESS, W.A. Recognition of health in hazards in industry: A review of materials and processes. 2.ed. New York, John Wiley \& Sons, 1995. 537p.

BUSCHLE, B.N.; PALMEIRO, J.K.; YOUSSEF BACILA SADE, Y.B.; MELO, V.F.; ANDRADE, M.G \& BATISTA, A.H. Cinética de liberação de chumbo de solos de área de mineração e metalurgia de metais pesados. R. Bras. Ci. Solo, 34:1865-1874, 2010.

CAMARGO, M.S.; ANJOS, A.R.M.; ROSSI, C. \& MALAVOLTA, E. Adubação fosfatada e metais pesados em Latossolo cultivado com arroz. Sci. Agric., 57:513-518, 2000.

CASALI, C.A.; MOTERLE, D.F.; RHEINHEIMER, D.S.; BRUNETTO, G.; CORCINI, A.L.M.; KAMINSKI, J. \& MELO, G.W.B. Formas e dessorção de cobre em solos cultivados com videira na Serra Gaúcha do Rio Grande do Sul. R. Bras. Ci. Solo, 32:1479-1487, 2008.

CONSELHO NACIONAL DO MEIO AMBIENTE - CONAMA Resolução $\mathrm{n}^{\circ}$ 420, de 28 de dezembro de 2009, 2009.

CORSI, A.C. \& LANDIM, P.M.B. Fluvial transport of lead, zinc and copper contents in polluted mining regions. Environ. Geol., 41:833-841, 2002.

CUNHA, F.G. Contaminação humana e ambiental por chumbo no vale do Ribeira, nos estados de São Paulo e Paraná, Brasil. Campinas, Universidade Estadual de Campinas, 2003. 83p. (Tese de Doutorado)

EMPRESA BRASILEIRA DE PESQUISA AGROPECUÁRIA EMBRAPA. Manual de métodos de análise de solo. 2.ed. Rio de Janeiro, 1997. 212p.

EYSINK, G.G.J. Metais pesados no Vale do Ribeira e em Igunde-Cananéia. R. CETESB Tecnol., 2:6-13, 1988.

GOLOBOÈANIN, D.D.; ŠKRIBÆ, B.D. \& MILJEVIÆ, N.R. Principal component analysis for soil contamination with PAHs. Chemom. Intellig. Lab. Syst., 72:219-223, 2004.

GOMES, P.C.; FONTES, M.P.F.; COSTA, L.M. \& MENDONÇA G.S. Extração fracionada de metais pesados em Latossolo Vermelho Amarelo. R. Bras. Ci. Solo, 21:543-551, 1997.

GUIMARÃES, V. Resíduos de mineração e metalurgia: Efeitos poluidores em sedimentos e em espécie biomonitora Rio Ribeira do Iguape-SP. São Paulo, Universidade de São Paulo, 2007. 160p. (Tese de Doutorado)

KEDE, M.L.F.M.; MOREIRA, J.C.; MAVROPOULOS, E.; ROSSI, A.M.; BERTOLINO, L.C.; PEREZ, D.V. \& ROCHA, N.C.C. Estudo do comportamento do chumbo em latossolos brasileiros tratados com fosfatos: contribuições para a remediação de sítios contaminados. Quim. Nova, 31:579-584, 2008. 
KIM, N.D. \& HILL, S.J. Sorption of lead and thallium on borosilicate glass and polypropylene: Implications for analytical chemistry and soil science. Environ. Technol., 14:1015-1026, 1993

KOTAS, J. \& STASICKA, Z. Chromium occurrence in the environment and methods of its speciation. Environ. Pollution, 107:263-283, 2000.

KUMMER, L. Mineralogia e fracionamento de metais pesados em solos de área de mineração e processamento de chumbo, Adrianópolis (PR). Curitiba, Universidade Federal do Paraná, 2008. 140p. (Tese de Mestrado)

KUMMER, L.; MELO, V.F.; BARROS, Y. \& AZEVEDO, J.C. Uso da análise de componentes principais para agrupamento de amostras de solos com base na granulometria e em características químicas e mineralógicas. Sci. Agr., 11:469-480, 2010.

LIM, C.H. \& JACKSON, M.L. Dissolution for total elemental analysis. In: PAGE, A.L., ed. Methods of soil analysis: Chemical and microbiological poperties. Madison, American Society of Agronomy, 1986. Parte 2. p.1-12.

MANN, S.S. \& RITCHIE, G.S.P. The influence of $\mathrm{pH}$ on the forms of cadmium in four west australian soils. Austr. J. Soil Res., 31:255-270, 1993.

MATTIAZZO, M.E.; BERTON, R.S. \& CRUZ, M.C.P Disponibilidade e avaliação de metais pesados potencialmente tóxicos. In: FERREIRA, M.E.; CRUZ, M.C.P.; RAIJ, B.van \& ABREU, C.A., ed. Micronutrientes e elementos tóxicos na agricultura. Jaboticabal, CNPq, Fapesp, Potafos, 2001. p. 222.

MELO, V.F.; FONTES, M.P.F.; NOVAIS, R.F.; SINGH, B. \& SCHAEFER, C.E.G.R. Características dos óxidos de ferro e de alumínio de diferentes classes de solos. R. Bras. Ci. Solo, 25:19-32, 2001.

MELO, V.F.; SCHAEFER, C.E.G.R.; NOVAIS, R.F.; SINGH, B. \& FONTES, M.P.F. Potassium and magnesium in clay minerals of some Brazilian soil as indicated by a sequential extraction procedure. Comm. Soil Sci. Plant Anal., 33:22032225,2002

MELO, V.F.; CORRÊA, G.F.; MASCHIO, P.A.; RIBEIRO, A.N \& LIMA, V.C. Importância das espécies minerais no potássio total da fração argila de solos do Triângulo Mineiro. R. Bras. Ci. Solo., 27:807-819, 2003.

MOITA NETO, J.M. \& MOITA, G.C. Uma introdução à análise exploratória de dados multivariados. Quim. Nova, 21:467. 469,1998

MORAES, R.P. Transporte de chumbo e metais associados no Rio Ribeira de Iguape, São Paulo, Brasil. Campinas, Universidade Estadual de Campinas, 1997. 94p. (Tese de Mestrado)

OLIVEIRA, M.A.F.; MANIESI, V.; TEIXEIRA, W. \& DAITX, E.C. Caracterização Isotópica de Metabasitos e Anfibolitos dos Grupos Açungui e Setuva na Porção Sul da Faixa Ribeira. Geol. USP Ser. Cient., 2:161-170, 2002
PANDOLFO, C.M.; CERETTA, C.A.; MASSIGNAM, A.M.; VEIGA, M. \& MOREIRA, I.C.L. Análise ambiental do uso de fontes de nutrientes associados a sistemas de manejo do solo. R. Bras. Eng. Agríc. Amb., 12:512-519, 2008.

RAKASASALAYA, M.; LANGDON, A.G. \& KIM, N.D. Assessment of the extend of lead distribution during sequential extraction by two different methods. Anal. Chim. Acta, 332:1-14, 1996.

SAHUQUILLO, A.; LOPEZ-SANCHEZ, J.F.; RUBIO, R.; RAURET, G.; THOMAS, R.P.; DAVIDSON, C.M. \& URE, A.M. Use of a certified reference material for extractable trace metals to assess sources of uncertainly in the BCR three-stage sequential extraction procedure. Anal. Chim. Acta, 382:317-327, 1999.

SANTOS, J.S.; SANTOS, M.L.P. \& OLIVEIRA, E. Estudo da mobilização de metais e elementos traços em ambientes aquáticos do semiárido brasileiro aplicando análises de componentes principais. Quim. Nova, 31:1107-1111, 2008.

SHAN, X.Q. \& CHEN, B. Evaluation of sequential extraction for speciation of trace metals in model soil containing natural minerals and humic acid. Anal. Chem., 65:802807, 1993

SHIOWATANA, J.; TANTIDANAI, N.; NOOKABKAEW, S. \& NACAPRICHA, D.A. Novel continuous-flow sequential extraction procedure for metal speciation in solids. J. Environ. Qual., 30:1195-1205, 2001.

SHUMAN, L.M. Zinc, manganese and copper in soil fractions. Soil Sci., 127:10-17, 1979.

SIMS, J.T. Soil $\mathrm{pH}$ effects on the distribution and plant availability of manganese, copper, and zinc. Soil Sci. Soc. Am. J., 50:367-373, 1986.

SPOSITO, G.; LUND, L.J. \& CHANG, A.C. Trace metal chemistry in arid-zonefield soils amended with sewage sludge: I. Fractionation of $\mathrm{Ni}, \mathrm{Cu}, \mathrm{Zn}, \mathrm{Cd}$, and $\mathrm{Pb}$ in solid phases. Soil Sci. Soc Am. J., 46:260-264, 1982.

STAHL, R.S. \& JAMES, B.R. Zinc sorption by iron-oxidecoated sand as a function of pH. Soil Sci. Soc. Am. J., $55: 1287-1290,1991$.

SUN, B.; ZHAO, F.J.; LOMBI, E. \& McGRATH, S.P. Leaching of heavy metals from contaminated soils using EDTA. Environ. Pollut., 113:111-120, 2001.

TESSIER, A.; CAMPBELL, P.G.C. \& BISSON, M. Sequential extraction procedure for the speciation of particulate trace metals. Anal. Chem., 51:844-851, 1979.

TU, Q.; SHAN, X.Q.; QIAN, J. \& NI, Z.M. Trace metal redistribution during extraction of model soils by acetic acid/sodium acetate. Anal. Chem., 66:3562-3598, 1994.

VALLADARES, G.S.; GOMES, E.G.; MELLO, J.C.C.B.S.; PEREIRA, M.G.; ANJOS, L.H.C.; EBELING, A.G. \& BENITES, V.M. Análise dos componentes principais e métodos multicritério ordinais no estudo de organossolos e solos afins. R. Bras. Ci. Solo, 32:285-296, 2008. 
YAMASHITA, D.M. Mobilidade de arsênio e metais pesados em solos do Vale do Ribeira, Iiporanga, SP. Campinas, Universidade Estadual de Campinas, 2004. 69p. (Tese de Mestrado)

ZHANG, M.; ALVA, A.K.; LI, Y.C. \& CALVERT, D.V. Chemical association of $\mathrm{Cu}, \mathrm{Zn}, \mathrm{Mn}$ and $\mathrm{Pb}$ in selectec sandy citrus soils. Soil Sci., 162:181-188, 1997.
WOWK, G.I.T. \& MELO, V.F. Avaliação do nível de chumbo, em solo de várzea, proveniente da reciclagem de bactérias. R. Bras. Eng. Agr. Amb., 9:613-622, 2005. 